\title{
Soluble Infinite-Range Model of Kinetic Roughening
}

\author{
M. Marsilit and A. J. Bray \\ Department of Physics and Astronomy, The University, Manchester M13 9PL, UK
}

(February 6, 2018)

\begin{abstract}
A modified Kardar-Parisi-Zhang (KPZ) equation is introduced, and solved exactly in the infinite-range limit. In the low-noise limit the system exhibits a weak-to-strong coupling transition, rounded for non-zero noise, as a function of the KPZ non-linearity. The strong-coupling regime is characterised by a double-peaked height distribution in the stationary state. The nonstationary dynamics is quite different from that of the stationary state.

05.40.+j, 64.60.Ht, 05.70.Ln
\end{abstract}

Typeset using REVTEX 
The field of kinetic roughening of surfaces has seen an explosion of interest in recent years (see, e.g, [1,2]). Numerous theoretical models have been proposed, of which the simplest non-trivial example is the Kardar-Parisi-Zhang (KPZ) equation [3]

$$
\partial_{t} h=\nu \nabla^{2} h+\lambda(\vec{\nabla} h)^{2}+\eta
$$

where $h(\vec{x}, t)$ specifies the height of the surface at point $\vec{x}$ at time $t$, and $\eta(\vec{x}, t)$ is a zero mean Gaussian noise with correlator $\langle\eta(\vec{x}, t) \eta(\vec{x}, t)\rangle=2 D_{0} \delta^{d}(\vec{x}-\vec{x}) \delta(t-t)$. This same equation is believed to describe the statistics of directed polymers in a random medium [4. [1], where $h$ has the meaning of a free energy.

For spatial dimensionality $d>2$, the KPZ equation is known to exhibit, for fixed noise strength $D_{0}$, a phase transition at $\lambda=\lambda_{c}$ from a 'weak-coupling' or 'smooth' phase for $\lambda<\lambda_{c}$ to a 'strong-coupling' or 'rough' phase for $\lambda>\lambda_{c}$. For $d \leq 2$, only the strong-coupling phase exists for any $\lambda>0$.

Despite intense effort in recent years, the properties of the strong-coupling phase are rather poorly understood. In the present Letter, we attempt to rectify this by presenting an exact solution of the model in the infinite range (or infinite $d$ ) limit. It turns out, however, that (四) has no stationary state in this limit, exhibiting instead a finite-time singularity. By adding an extra, physically-motivated term [see (四) below], this deficiency is remedied and a stationary state obtained.

The main properties of the model are: (i) In the limit $D \rightarrow 0^{+}$, there is a sharp phase transition at a critical coupling $g_{c}$ from a smooth weak-coupling phase to a rough strongcoupling phase; (ii) The strong-coupling phase has a two-peaked height distribution in the stationary state, with the height difference depending on $g$ but not on $t$; (iii) At finite noise strength the transition is rounded, but the qualitative features of the weak and strongcoupling regimes are very different; (iv) The dynamics of the approach to the stationary state is quite different from the dynamics of correlations in the stationary state, contrary to the assumptions of conventional scaling theories.

We shall adopt an approach which parallels the one used in mean-field, infinite range, 
theories for equilibrium statistical mechanics (e.g. in the Sherrington-Kirkpatrick model of spin glasses [5]), introduced in this context originally by Luck and Orland [6]. This amounts to assuming, once eq. (11) is discretized on a lattice, that every site is the nearest neighbor of every other site. Therefore the number $z$ of nearest neighbors of each site equals the system size. For Euclidean lattices $z=2 d$, so this approximation is expected to be valid for $d \gg 1$.

Let $h_{i}(t) \equiv h\left(\vec{x}_{i}, t\right)$ where $\vec{x}_{i}$ is the $i^{\text {th }}$ lattice point and let $\sqrt{z}$ be the mesh length of the lattice discretization. Let $\langle h\rangle_{t}=\frac{1}{z} \sum_{i=1}^{z} h_{i}(t)$ be the average height at time $t$; then $\phi_{i}(t)=h_{i}(t)-\langle h\rangle_{t}$ is the local height fluctuation on site $i$. The discretized form of the Laplacian is $\left.\nabla^{2} h\right|_{\vec{x}_{i}}=-\phi_{i}$, while the nonlinear term becomes $\left.(\vec{\nabla} h)^{2}\right|_{\vec{x}_{i}}=\left(\left\langle\phi^{2}\right\rangle_{t}+\phi_{i}^{2}\right) / 2$. After subtracting the equation $\partial_{t}\langle h\rangle_{t}=\lambda\left\langle\phi^{2}\right\rangle_{t}$ for the mean height, (1) becomes

$$
\partial_{t} \phi=-\nu \phi+\lambda\left(\phi^{2}-\left\langle\phi^{2}\right\rangle_{t}\right) / 2+\zeta
$$

where the site subscript has been suppressed. Here the noise, in view of our choice of the lattice discretization, satisfies $\left\langle\zeta(t) \zeta\left(t^{\prime}\right)\right\rangle=2 D \delta\left(t-t^{\prime}\right)$ with $D=D_{0} z^{-d / 2}$.

That (21) leads to difficulties at large $t$ can be understood by expressing it in the form of a Langevin equation,

$$
\partial_{t} \phi=-V^{\prime}(\phi)+\eta
$$

(here and below the prime denotes a derivative) and noting that the potential $V$ is unbounded from below as $\phi \rightarrow \infty$. This suggests that there is no stationary state, and that a finite-time singularity occurs. The 'self-consistent' time dependence of $V$ which comes from the $\left\langle\phi^{2}\right\rangle_{t}$ term in (2) make things more complex. Numerical integration of the Langevin equation (evolving an ensemble of $z \gg 1$ systems simultaneously to compute $\left\langle\phi^{2}\right\rangle_{t}$ ) confirm the conclusion of a finite time singularity. This suggests that the $d \rightarrow \infty$ limit of the KPZ equation is, as it stands, not well defined.

Since the KPZ equation is usually obtained from a small gradient expansion, it is reasonable to try to remedy to the failure of eq. (2) by including a further term. In order to do this we need to specialize to a particular model. Indeed while the terms appearing in eq. 
(四) belong to the small gradient expansion of a large number of models, which is the origin of the assumed KPZ universality, the first neglected term differs from model to model. For example, in the small gradient expansion for directed polymers, the next term in (1) would be $\kappa\left(\nabla^{2} h\right)^{2}$. On the contrary, in models for growing interfaces the next term is proportional to $(\vec{\nabla} h)^{2} \nabla^{2} h[0]$. Focusing on the latter models, in the following, we will discuss the equation

$$
\partial_{t} h=\nu \nabla^{2} h+\lambda(\vec{\nabla} h)^{2}+\kappa(\vec{\nabla} h)^{2} \nabla^{2} h+\eta
$$

Grouping the first and the third term in the right hand side of eq. (4), this reduces to eq. (11) with a space dependent coefficient $\nu$ which is larger on steep portions of the interface $(\kappa>0)$. This is appropriate for restricted solid-on-solid (RSOS) models [1] for growing interfaces, where, for entropic reasons, the fluctuations are suppressed faster by the constraint on more inclined surface elements [7]. The second term also results from the effect the constraint has on the growth mechanism. Incoming particles are more likely to stick to the interface, causing it to grow, on flatter parts of the surface than on inclined ones [7,8]. Eq. (4) includes both these effects of the constraint to the same (second) order in their gradient expansion [9.

Let us now discuss the infinite range limit of eq. (雨) for $\kappa>0$. Performing the same steps as before leads to

$$
\partial_{t} \phi=\mu_{t}-\left(1+s_{t}\right) \phi+g \phi^{2}-\phi^{3}+\zeta
$$

Here we have set $\nu=\kappa=1$ via the transformations $t \rightarrow t / \nu$ and $\phi \rightarrow \phi \sqrt{\nu / \kappa}$. Accordingly $g=\lambda /(2 \sqrt{\nu \kappa})$ and $D=D_{0} \kappa \nu^{-2} z^{-d / 2}$, where $D_{0}$ is the strength of the noise in eq. (幽. Furthermore in this equation we have defined the (time-dependent) parameters

$$
\mu_{t}=\left\langle\phi^{3}\right\rangle_{t}-g\left\langle\phi^{2}\right\rangle_{t} \quad \text { and } \quad s_{t}=\left\langle\phi^{2}\right\rangle_{t}
$$

which have to be determined self-consistently. The parameter $\mu_{t}$ enforces the condition $\langle\phi\rangle_{t}=0$ for all $t$. The interface velocity is $v_{t}=\partial_{t}\langle h\rangle_{t}=g s_{t}-\mu_{t}$.

Eq. (5) can be easily put in the form (3) with a time-dependent potential 


$$
V_{t}(\phi)=-\mu_{t} \phi+\left(1+s_{t}\right) \frac{\phi^{2}}{2}-g \frac{\phi^{3}}{3}+\frac{\phi^{4}}{4}
$$

which is now bounded from below.

If we assume the parameters $\mu$ and $s$ approach finite limits as $t \rightarrow \infty$, then the stationary state is given by

$$
P_{t=\infty}(\phi) \propto \exp \left[-V_{\infty}(\phi) / D\right]
$$

On the other hand, the values of $\mu_{\infty}$ and $s_{\infty}$ which enter the potential $V_{\infty}$ are obtained from eq. (6) from the distribution $P_{\infty}(\phi)$. A stationary state exists if one can find $\mu_{\infty}(g, D)$ and $s_{\infty}(g, D)$ which solve self-consistently equations (6, 7) and (8).

Let us consider first the limit $D \rightarrow 0^{+}$. For small $g$ the potential $V_{\infty}$ has a single minimum at $\phi^{-}=0$, giving $s_{\infty}=0=\mu_{\infty}$. For $g>\sqrt{3}$, the potential has a pair of inflection points and for $g>2$ a second minimum at $\phi^{+}=\frac{1}{2}\left(g+\sqrt{g^{2}-4}\right)$ appears. However, for $g<3 / \sqrt{2}$ this minimum has a higher potential than the minimum at $\phi=0$, so its occupation is exponentially suppressed with respect to that of the other minimum for $D \rightarrow 0^{+}$, and $s_{\infty}$ remains zero (in fact $s_{\infty}=O(D)$ for $D$ small). It is only when, increasing $g, V_{\infty}\left(\phi^{+}\right)-V_{\infty}\left(\phi^{-}\right)$ becomes of order $D$ that the weight of the + minimum becomes comparable to that of the - one. In the limit $D=0^{+}$this happens at $g_{c}\left(0^{+}\right)=3 / \sqrt{2}=2.12132 \ldots$ For $g>g_{c}$ both minima contribute to $\left\langle\phi^{2}\right\rangle_{\infty}$ so that $s_{\infty}$ starts to increase with $g$ even for $D=0^{+}$. Since both minima must have non-vanishing occupation to ensure $\langle\phi\rangle_{\infty}=0, s_{\infty}$ and $\mu_{\infty}$ adjust their values such that both minima have the same depth. This implies that $V_{\infty}(\phi)=\bar{V}+\frac{1}{4}\left(\phi-\phi^{-}\right)^{2}\left(\phi-\phi^{+}\right)^{2}$ where $\phi^{ \pm}$are given by $V_{\infty}^{\prime}\left(\phi^{ \pm}\right)=0$. Moreover, in this limit $P_{\infty}(\phi)=\alpha \delta\left(\phi-\phi^{-}\right)+(1-\alpha) \delta\left(\phi-\phi^{+}\right)$. Using $\langle\phi\rangle_{\infty}=0$ and evaluating $\left\langle\phi^{2}\right\rangle_{\infty}$ one finds $s_{\infty}=-\phi^{-} \phi^{+}$. The solution to these equations yields $\phi^{ \pm}=\frac{1}{3} g \pm \sqrt{\frac{2}{9} g^{2}-\frac{1}{2}}$ and

$$
s_{\infty}\left(g, 0^{+}\right)=\frac{g^{2}}{9}-\frac{1}{2}, \quad \mu_{\infty}\left(g, 0^{+}\right)=-\frac{g}{3}\left(\frac{g^{2}}{9}-\frac{1}{2}\right)
$$

for $g>g_{c}$. The potential at the bottom of both minima is $\bar{V}=-s_{\infty}^{2} / 4$. These minima are separated by a maximum at $\phi^{o}=g / 3$ with $V_{\infty}\left(\phi^{o}\right)=\frac{1}{108} g^{2}\left(g^{2}-3\right)$. 
The emerging picture for $D=0^{+}$is that of a sharp phase transition at $g_{c}$. This separates physically different weak-coupling $\left(g<g_{c}\right)$ and strong-coupling $\left(g>g_{c}\right)$ regimes in which the static and dynamic properties differ substantially. Note that $D=0^{+}$means we are taking the limit $D \rightarrow 0$ after the limit $t \rightarrow \infty$, in order to establish a stationary state.

Before commenting on the nature of the two phases, it is worth discussing the behavior in the critical region at nonzero noise. This is available if one includes the gaussian fluctuations in the saddle point calculation of eqs.(6). If one defines $g_{c}(D)=\frac{3}{\sqrt{2}}\left(1+\frac{D}{2} \ln \frac{D}{2}\right)$ and $\epsilon \equiv g-g_{c}$, the result can be cast in the scaling form $s_{\infty}=D f(\epsilon / D)$, for $\epsilon$ and $D$ both small, where $f(x)$ satisfies $3(1+f+\ln \sqrt{f-1})=\sqrt{2} x$. A similar scaling form holds for $\mu_{\infty}=-\sqrt{2} D-s_{\infty} / \sqrt{2}$. For $D>0$ the transition is smoothed and a crossover between the two regimes occurs. We checked by a numerical integration of the Langevin eq. (5) for $\sim 10^{6}$ realizations (see fig. 1) that the stationary state discussed so far is the one which is actually reached by the dynamics.

Let us discuss our findings in the framework of kinetic roughening. The parameter $s$ has the meaning of the square of the interface width $s=\left\langle(h-\langle h\rangle)^{2}\right\rangle$. Its behavior with the linear system size $L$, in the stationary state, defines the roughness exponent $\chi: s_{\infty}(L) \sim L^{2 \chi}$. In our case, we considered from the beginning an infinite system, so a finite value of $s_{\infty}$, both in the weak and in the strong coupling phases, implies $\chi=0$. This agrees with the result found for directed polymers in random media in the $d \rightarrow \infty$ limit [10]. However, the nature of the stationary state differs dramatically. Indeed eq. (8) predicts, for the strong coupling regime, a double peaked distribution in contrast to the highly asymmetric but single peak distribution found for directed polymers [11]. The most striking feature of the distribution (8) is that it predicts the presence of bumps of a definite height $\phi^{+}-\phi^{-}$in the surface for $g>g_{c}$. Moreover, the minima of the potential are separated by a barrier $\Delta V \simeq(g / 3)^{4}$ which is very large for $g \gg 1$. This suggests very large relaxation times $\tau \sim \exp (\Delta V / D)$ in the stationary state. The numerical simulation of eq. (5) indeed confirms this conclusion (see fig. (1). The interface velocity, $v_{\infty}=g s_{\infty}-\mu_{\infty}$, also differs in the two regimes, being $O(D)$ for $g<g_{c}$ and $O\left(g-g_{c}\right)$ for $g>g_{c}$. 
Let us now discuss the non-stationary dynamics in the strong-coupling regime. Consider the Langevin dynamics of an ensemble of a $z \gg 1$ system $\left\{\phi_{i}\right\}$ starting from a flat substrate: $P_{t=0}(\phi)=\delta(\phi)$. Therefore $s_{0}=0=\mu_{0}$. It is convenient to think again in terms of the potential (7). Initially this has two minima, but only the minimum at $\phi_{0}^{-}=0$ is occupied, while the one at $\phi_{0}^{+} \simeq g$, which is very deep, with $V_{0}\left(\phi^{+}\right) \simeq-g^{4} / 12$, is empty. As time goes on, the locations of the minima, which are given by $V_{t}^{\prime}\left(\phi_{t}^{ \pm}\right)=0$, adjust according to the running values of $s_{t}$ and $\mu_{t}$. At the same time, the potential barrier between the two minima increases. After an initial transient, the distribution of the ensemble $\left\{\phi_{i}\right\}$ can be well approximated by $P_{t}(\phi)=\alpha_{t} \delta\left(\phi-\phi_{t}^{+}\right)+\left(1-\alpha_{t}\right) \delta\left(\phi-\phi_{t}^{-}\right)$. This allows one to transform the equations $\langle\phi\rangle_{t}=0$ and $s_{t}=\left\langle\phi^{2}\right\rangle_{t}$ in the form $\alpha_{t}=-\phi_{t}^{-} /\left(\phi_{t}^{+}-\phi_{t}^{-}\right)$and $s_{t}=-\phi_{t}^{-} \phi_{t}^{+}$. These relations leave only one independent variable, whose dynamics is given by

$$
\frac{d \alpha}{d t} \simeq \exp \left[-\frac{V_{t}\left(\phi_{t}^{o}\right)-V_{t}\left(\phi_{t}^{-}\right)}{D}\right]
$$

This equation describes the noise activated processes $\phi^{-} \rightarrow \phi^{+}$across the potential barrier. It neglects the backward processes $\phi^{+} \rightarrow \phi^{-}$. It is therefore valid as long as $V_{t}\left(\phi^{-}\right)-$ $V_{t}\left(\phi^{+}\right) \gg D$. Since the scale of $V_{t}$ is of order $g^{4} \gg D$, this is a good approximation for times for which the system is not too close to the stationary state.

The equation (10) cannot be solved exactly. However some progress can be made assuming $\alpha \ll 1$, and considering the leading behavior in $\alpha$ : $\phi^{+}=g(1-\alpha), \phi^{-} \simeq-g \alpha$ and $\phi^{o} \simeq 2 g \alpha$. To leading order in $\alpha \ll 1$, after some algebra, one finds

$$
\frac{V_{t}\left(\phi_{t}^{o}\right)-V_{t}\left(\phi_{t}^{-}\right)}{D}=\frac{9 g^{4} \alpha^{3}}{2 D}=\left(\frac{\alpha}{t_{c}}\right)^{3}
$$

where the characteristic time $t_{c}=\left(\frac{2 D}{9 g^{4}}\right)^{1 / 3}$ has been defined. Note that in the stationary state $\alpha_{\infty} \rightarrow 0.1464 \ldots$ as $g \rightarrow \infty$. Therefore the leading order in $\alpha_{t}$ can give a reasonable approximation even for late times.

For $\alpha_{t} \ll t_{c} \ll 1$, the saddle point approximation to $P_{t}(\phi)$ becomes questionable. In the regime $t_{c} \ll \alpha_{t} \ll 1$, where we expect our approximations to be reasonable, eq. (10), to leading order in $\alpha_{t}$, predicts a behavior $\alpha_{t} \sim(\ln t)^{1 / 3}$. The "interface width" $s_{t}$ can easily be 
related to $\alpha_{t}$. Within our approximations $\left(g^{4} \gg D\right.$ and $\left.\alpha_{t} \ll 1\right)$ one finds $s_{t} \simeq D+\left(\phi^{+}\right)^{2} \alpha_{t}$ to leading order in $\alpha_{t}$. Therefore our previous discussion suggests

$$
s_{t} \sim(\ln t)^{1 / 3} .
$$

Figure 1 shows that for $g=3$ and $D=0.0625$ eq. (11) yields an accurate fit of the simulation results.

This regime holds in general for $D \ll g^{4}$ (when the saddle point approximation is valid) and for intermediate times. When the backward processes $\phi^{+} \rightarrow \phi^{-}$becomes important $\left(V\left(\phi^{-}\right)-V\left(\phi^{+}\right) \sim D\right)$, eq. (10) ceases to hold. The system starts to relax to the stationary state (see figure [1).

It is important to note that the 'off equilibrium' dynamics has no relation whatsoever with the stationary dynamics. In particular we found that the relaxation times to the stationary state are orders of magnitude smaller than the ones which characterizes the dynamics in the stationary state. In the case illustrated in figure \&, for example, the fit of the approach to the stationary state yields a characteristic time $\approx 340$, whereas the correlation function in the stationary state decays over characteristic times of order $\exp (\Delta V / D) \approx 8000$. The scaling relation usually assumed to relate the early stages of growth to the stationary state behavior [1] fails dramatically. Finally we observed that the time scales involved in the dynamics of the model, even for moderate values of $g$ and $D$, can well be beyond the reach of numerical simulation (already for $g=4$ and $D=0.125$ the time to reach the stationary state is of order $10^{5}$ ).

In conclusion, we have discussed a high-dimensional limit for growth equations. We have shown that this limit is plagued by finite time singularities for the KPZ equation as it stands. We have modified the model by including a further term in the small gradient expansion of the equation for RSOS growth models. Even though this term would be irrelevant, according to the usual folklore based on dimensional analysis, we have shown that it allows for a well defined and non-trivial high dimensional limit. In particular, in the stationary state the

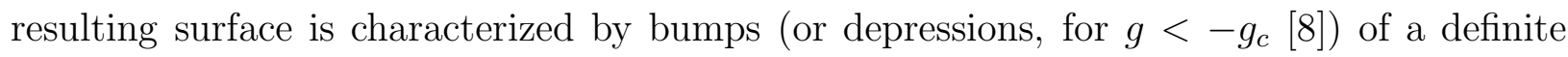


height in the strong-coupling regime. Although the analysis has been carried out explicitly in the limit of vanishing noise, $D=0^{+}$, the same qualitative picture obtains for $D>0$, with the height distribution changing (but now smoothly) from single-peaked to double-peaked with increasing $g$.

This strong-coupling stationary state differs substantially from the one of directed polymers in random media. Our results suggest that the mapping discussed in ref. [12] between RSOS models and directed polymers may fail in high dimensions. One might wonder whether the scenario outlined so far is specific to eq. (4) or whether it applies in a more general class of models. Note, in this respect, that the sign of $\kappa$ plays a very important role. For $\kappa<0$ the infinite dimensional limit is plagued by finite time singularities like those found for the KPZ equation. Actually, eq. (4) with $\kappa<0$ would result from the small gradient expansion of the equation for the Eden model or for ballistic aggregation [7]. On the other hand, for other generalizations of the KPZ equation (like e.g. the one used in ref. [13]) in the spirit of RSOS models, we found the same scenario as in this work [14. These observations raise the question of whether a KPZ universality class exists at all in high enough dimensions. It is interesting to note that already for $d=2$ there are discrepancies between the exponents obtained by numerical simulations for different models [15]. It is also noteworthy that already in $d=2$ finite-time singularities in the simulation of the KPZ equation have been observed [16].

If the behavior discussed above applies to any large but finite dimension, a second key question is what is the dimension $d_{c}$ above which this behavior sets in. In this respect we note that finite height bumps and structures with very large relaxation times have been observed [17] in $d=2$ simulations of a regularized KPZ equation.

We finally point out that the existing numerical results on RSOS models [18] in high dimensions do not completely rule out the possibility that the behavior we have discussed sets in for relatively low dimensions. Indeed in ref. [18 only the early stages of growth were investigated and an exponent was extracted under the assumption of dynamic scaling. Our model shows that this assumption is far from trivial. Furthermore, in a numerical simulation 
for the interface thickness it might be quite hard to disentangle a power law with a small power from a behavior like that of eq. (11).

We thank T. Blum, M. A. Moore, and T. J. Newman for discussions. This work was supported by EPSRC grant GR/H27496. 


\section{REFERENCES}

* Present address: Institut de Physique Théorique, Université de Fribourg, Pérolles CH1700 Fribourg.

[1] T. Halpin-Healy and Y-C. Zhang, Phys. Rep. 254, (1995).

[2] J. Krug, Adv. Phys., to appear.

[3] M. Kardar, G. Parisi, and Y-C. Zhang, Phys. Rev. Lett. 56, 889 (1986).

[4] M. Kardar and Y-C. Zhang, Phys. Rev. Lett. 58, 2087 (1987).

[5] D. Sherrington and S. Kirkpatrick, Phys. Rev. Lett. 35, 1792 (1975).

[6] J. M. Luck and H. Orland, unpublished.

[7] M. Marsili, A. Maritan, F. Toigo and J. R. Banavar, preprint (1995), submitted to Rev. Mod. Phys; A. Maritan, F. Toigo, J. Koplik and J. R. Banavar, Phys. Rev. Lett. 69, 3193 (1992).

[8] For RSOS models, $\lambda<0$. The invariance of (四) under $\lambda \rightarrow-\lambda, h \rightarrow-h$, implies however that the sign of $\lambda$ is unimportant, and we take $\lambda>0$ throughout.

[9] The $\lambda$ term is a correction to a constant $f$ term, which can be eliminated by a Galilean transformation.

[10] B. Derrida and H. Spohn, J. Stat. Phys. 51, 817 (1988).

[11] J. Cook and B. Derrida: J. Stat. Phys. 63, 505 (1991).

[12] See e.g. L-H. Tang, B.M. Forrest and D.E. Wolf Phys. Rev. A 45, 7162 (1995).

[13] S.E. Esipov, Phys. Rev. E 51, 4369 (1995).

[14] The key stability requirement is that the potential $V(\phi)$ be bounded below.

[15] B.M. Forrest and L-H. Tang, Phys. Rev. Lett. 64, 1405 (1990) (RSOS model); J.M. 
Kim, M.A. Moore and A.J. Bray, Phys. Rev. A 44, 2345 (1991) (directed polymers in random media); D. Y. K. Ko and F. Seno, Phys. Rev. E 50, R1741 (1994) (RSOS model and ballistic aggregation).

[16] J.P. Doherty, M.A. Moore, J.M. Kim and A.J. Bray, Phys. Rev. Lett. 72, 2041 (1994); J.M. Kim, private communication.

[17] T. Newman, private communication

[18] T. Ala-Nissila, T. Hjelt, J.M. Kosterlitz and O. Venäläinen, J. Stat. Phys. 72, 207 (1993). 


\section{FIGURES}

FIG. 1. Upper curve: Stationary state correlation function $C(t) \equiv\left\langle\phi\left(t+t_{0}\right) \phi\left(t_{0}\right)\right\rangle$ for a system with $g=3$ and $D=1 / 16$. The fit to $C(t)=A \exp (-t / \tau)$ yields $\ln \tau \simeq 8.97$ to be compared to $\Delta V / D=9$. Lower curve: behavior of $s_{t}$, starting from the flat configuration, for the same system. The fit was obtained plotting $s^{3} \simeq 0.015+0.017 \ln t$ versus $\ln t$. 


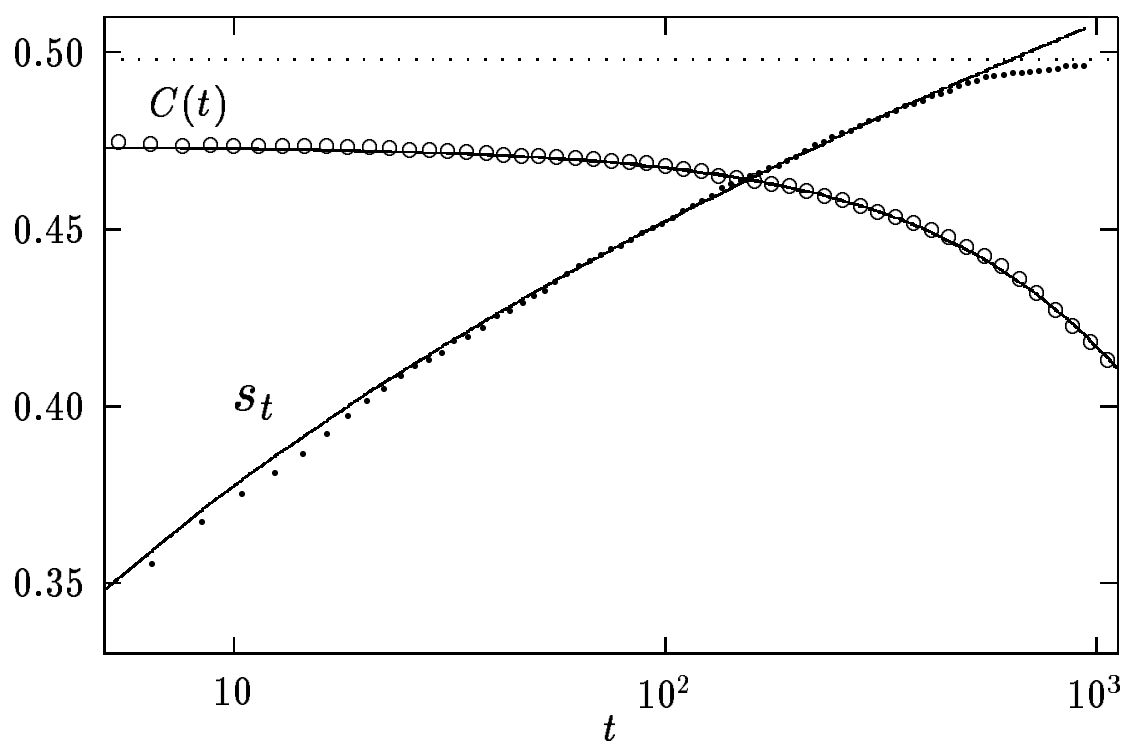

